\title{
Superconductivity in compressed hydrogen-rich materials: pressing on hydrogen.
}

\author{
Viktor V. Struzhkin* \\ Geophysical Laboratory, Carnegie Institution of Washington, \\ 5251 Broad Branch Road NW, Washington DC 20015, USA
}

Periodic table of elements starts with Hydrogen, a simplest element of all. The simplicity is lost when the element is compressed to high densities or participates in a chemical bonding in compounds, being subjected to "chemical pressure" of surrounding atoms or molecules. The chemical nature of hydrogen is dictated by its simplest electronic shell, which has only one electron. Hydrogen can donate this electron and behave like alkali metal, or accept an extra electron and form a hydride ion with closed shell resembling a group VII element. The complexity of hydrogen goes beyond these simplest configurations, when hydrogen is involved in a multicenter bonding or in hydrogen bonds. This complex behavior is tightly related to the ability of hydrogen to participate in the process of electronic transport in solids and potentially be able to contribute to the superconductivity in a material. Hydrogen by itself when compressed to immense pressures of 400-500 GPa may form a simple atomic phase with very high critical superconducting temperatures $\left(\mathrm{T}_{\mathrm{c}}\right)$ well above room temperature. While this theoretical insight awaits confirmation at pressures at the limit of current experimental capabilities, a variety of other hydrogen-rich materials have been suggested recently to have record high $\mathrm{T}_{\mathrm{c}}$ values. The very existence of many of these materials still lacks experimental confirmation. In this review article, we will present an extensive list of such predicted materials. We will also review superconductivity in classical hydrides (mostly metal hydrides) and current theoretical understanding of relatively low $\mathrm{T}_{\mathrm{c}}{ }^{ } \mathrm{s}$ in metal hydrides of transition and noble metals.

*Corresponding author:

Viktor Struzhkin

Tel.: +1 2024788952

Fax: +1 2024788901

Email: vstruzhkin@carnegiescience.edu 
Article outline

1. Introduction

2. Phonon-driven superconductivity in hydrides
A. Electron-phonon coupling and anharmonic effects
B. Anharmonic reduction of $\mathrm{T}_{\mathrm{c}}$ : PdH, PtH.

3. Hydrogen "doping" with other elements
A. Silane and its siblings
B. Polyhydrides of alkali and alkaline earth metals

4. Prospects for room temperature superconductor based on hydrogen-rich materials: concluding remarks

5. Appendix: Properties of the superconducting state of hydrides 


\section{Introduction}

As we may expect, chemical nature of hydrogen dictates its participation in transport phenomena in solids. "Dual" nature of hydrogen as an electron donor or acceptor, and its tendency to form multicenter bonds of various degree of complexity (1), provides for a variety of chemical environments for hydrogen (2). While "ionic" character of a hydride ion or a proton in most of these environments (3) is leading to insulating properties, a multicenter or metallic bonding (1) promotes materials with metallic conductivity. Within a BCS (Bardeen, Cooper and Schrieffer) scenario of superconductivity (4) high vibrational frequencies of hydrogen atoms raised hopes (5) for a high critical temperature in conducting hydrogen and hydrogen-rich materials (๑). However, multiple attempts to find a material with high critical superconducting temperature in hydrides of transition and rare-earth metals did not produce a high- $\mathrm{T}_{\mathrm{c}}$ material. The highest $\mathrm{T}_{\mathrm{c}}$ 's observed in hydrides of $\mathrm{Pd}$ and $\mathrm{Pd}-\mathrm{Au}, \mathrm{Pd}-\mathrm{Cu}$ alloys did not exceed temperatures of the order of $15-17 \mathrm{~K}(7,8)$. Moreover, substantial inverse isotope effect was observed in $\mathrm{PdH}$ and $\mathrm{PdD}$, at first raising doubts in the validity of the electronphonon coupling mechanism for superconductivity in these materials, and later leading to the understanding of the role played by anharmonicity of hydrogen vibrations in such "simple" hydrides (9). It should be noted that there are published claims of nearly roomtemperature superconductivity in $\mathrm{PdH}_{\mathrm{x}}$ materials (10), which have not been confirmed by other experimentalists and have no theoretical backing at the moment.

The ability to create hydrogen-based materials with desired properties has received tremendous boost recently from a variety of government funded programs aimed at a viable hydrogen storage material for automotive and energy-storage related applications 
$(11,12)$. Chemical compounds $(11)$, weakly interacting molecular materials $(12,13)$, surface interactions (14) have been attempted with limited success to conveniently store and retrieve hydrogen. However, no practical material for hydrogen storage did emerge from this massive effort so far. Despite the deceptive simplicity hydrogen turned out extremely complex chemical entity as a component of useful materials. The properties of hydrogen in high-pressure environment become even more complex. High energy density (15), high-temperature superconductivity and superfluidity (16) are expected in dense hydrogen and hydrides. Ambient pressure metastable metallic phase of hydrogen, consisting of weakly interacting chains of hydrogen atoms with interatomic distances about $1.06 \AA$ ' was predicted in 1970'ies by Brovman and Kagan (17). Ashcroft (6) proposed a metallic state in hydrogen-rich alloys, where the electronic bands of hydrogen and the host element(s) overlap. In the work that followed, the superconductivity was found with $\mathrm{T}_{\mathrm{c}}$ approaching $20 \mathrm{~K}$ in transport measurements performed by Eremets et al. (18). This claim was subjected to experimental (and theoretical) scrutiny as discussed in section 2 of this review. As a development of this idea, very unusual metallic polyhydrides of lithium have been proposed $(19,20)$ to exist at pressures about $100 \mathrm{GPa}$, well below the expected metallization pressure of pure hydrogen (21). Stable polyhydrides of alkali metals have been predicted recently by theoretical analysis of MHn ( $\mathrm{M}=\mathrm{Li}, \mathrm{Na}, \mathrm{K}, \mathrm{Rb}, \mathrm{Cs})$ compounds with variable hydrogen composition $(19,20,22-25)$. These alkali and alkali earth polyhydrides will be reviewed in section 3 .

Finally, we will discuss the prospects for high- $\mathrm{T}_{\mathrm{c}}$ hydrogen-rich materials in a highpressure environment. Both theoretical insights and experimental challenges will be discussed. 


\section{Phonon-driven superconductivity in hydrides}

\section{A. Electron-phonon coupling and anharmonic effects}

We give below a quick summary of the results for electron-phonon coupling driven superconductivity relevant for the discussion of hydrogen-based superconductors. The critical superconducting temperature for a phonon-mediated superconductor can be conveniently represented by modified McMillan formula $(26,27)$ :

$$
T_{\mathrm{c}}=\frac{\omega_{\log }}{1.2} \exp \left[-\frac{1.04(1+\lambda)}{\lambda-\mu^{*}(1+0.62 \lambda)}\right]
$$

where $\omega_{\log }$ is a logarithmic average of the phonon frequency, $\lambda$ is electron-phonon coupling constant, and $\mu^{*}$ is the Coulomb pseudopotential of Morel and Anderson (28).

Another useful formula is defining electron phonon coupling parameter $\lambda$

$$
\lambda \equiv \frac{N\left(\epsilon_{F}\right) a^{2}}{M \omega_{E}^{2}}
$$

in terms of the density of the electronic states $\mathrm{N}\left(\mathrm{E}_{\mathrm{F}}\right)$ at the Fermi level, the phonon frequency $\omega_{\mathrm{E}}$ and effective mass $\mathrm{M}$, and the Holstein parameter $\alpha$, a Fermi surface average of the electronic matrix element of the change in crystal potential as particular phonon mode-related atoms are moved (26). Partial contribution of phonon modes was analyzed in (29) for weakly coupled phonons; we show a figure with the results of this analysis (Fig.1) 
/E $\delta T_{c} / \delta \alpha^{2}(\Omega) F(\Omega)$

$\frac{\delta T_{c}}{{ }^{2}(\Omega) F(\Omega)} \equiv \lim _{\epsilon \rightarrow 0} \frac{\delta T_{c}}{\epsilon}=\frac{1}{1+\lambda} G(\bar{\Omega})$

ere

\section{1}

(21a

Fig.1. Function $\mathrm{G}(\bar{\Omega}), \quad \bar{\Omega}=\Omega / \mathrm{T}_{\mathrm{c}}$ is the reduced frequency $\left(\hbar=1, \mathrm{k}_{\mathrm{B}}=1\right)$. Reproduced from Solid State Commun. from Mitrović and Carbotte (29).

Fig.1 shows relative partial derivative of the $T_{c}$ with respect to small change in the partial coupling strength $\alpha^{2}(\Omega) \mathrm{F}(\Omega)$ at phonon frequency $\Omega$, with broad maximum around $\Omega=10$. It means that the phonon frequencies contributing mostly to $T_{c}$, are about factor of 10 higher in frequency than the $T_{c}$ (in equivalent units, $\hbar \omega / k_{B} T_{c}$ ). This result along with Eq.1, strongly favors high vibrational frequencies as a prerequisite of high $\mathrm{T}_{\mathrm{c}}$ values. The highest vibrational frequencies are found in hydrogen in hydrides; for this reason hydrogen-rich materials have attracted significant amount of attention in the search for highest $\mathrm{T}_{\mathrm{c}}$ values in materials. However, historically the quest for high- $\mathrm{T}_{\mathrm{c}}$ hydrogen based materials did not produce any significant results. The early era before high- $\mathrm{T}_{\mathrm{c}}$ cuprates, was reviewed in (30). Despite significant effort, only two families of relatively high $\mathrm{T}_{\mathrm{c}}$ materials have been found with $\mathrm{Th}(31)$ and $\mathrm{Pd}(7,32)$ hydrides. Both systems showed anomalous isotope effect when doped with deuterium; ThH(D) 3.64 did not show any isotope effect, which initially raised doubts regarding the phonon-mediated mechanism, and $\mathrm{PdH}(\mathrm{D})$ showed inverse isotope effect (9). In the theoretical and 
experimental papers that followed discovery of $\mathrm{PdH}$ superconductor, the anomalous isotope effect was understood as resulting from anomalously large anharmonicity of hydrogen (deuterium) vibrations $(33,34,35-37)$. While earlier works used empirically scaled experimental phonon spectrum (34), the latest theoretical efforts have explained the observed anharmonicity from the first principles (37). Since the anharmonic effects are not treated explicitly in lately predicted high- $\mathrm{T}_{\mathrm{c}}$ 's in transition and noble metal hydrides $(38,39)$, we will give a brief overview of current understanding of the anharmonicity effects on $\mathrm{T}_{\mathrm{c}}$ in these materials.

\section{B Anharmonic reduction of $\mathrm{T}_{\mathrm{c}}$ : $\mathrm{PdH}, \mathrm{PtH}$.}

In the early paper Klein et. al. (34) have used the measured phonon densities of states in $\mathrm{PdH}(\mathrm{D})$ (40) to introduce semiempirical correction to the electron-phonon coupling constant, and they concluded that observed (quartic) anharmonicity is sufficient to explain the anomalous isotope effect. Similar considerations were used in several other theoretical approaches; Klein and Cohen have calculated the phonon spectra and were able to reproduce the inverse isotope effect qualitatively (41). They also noticed strong dependence of the isotope effect on Coulomb pseudopotential (Eq. 1), which is theoretically justified (42). Anharmonic vibrations of hydrogen in $\mathrm{AlH}_{3}$ were suggested (43) as the reason for disagreement between "harmonic" calculation and experimental findings (44). In Fig. 2 we show highly anharmonic character of hydrogen vibrations (quartic anharmonicity) as follows from the frozen-phonon calculations (43). 

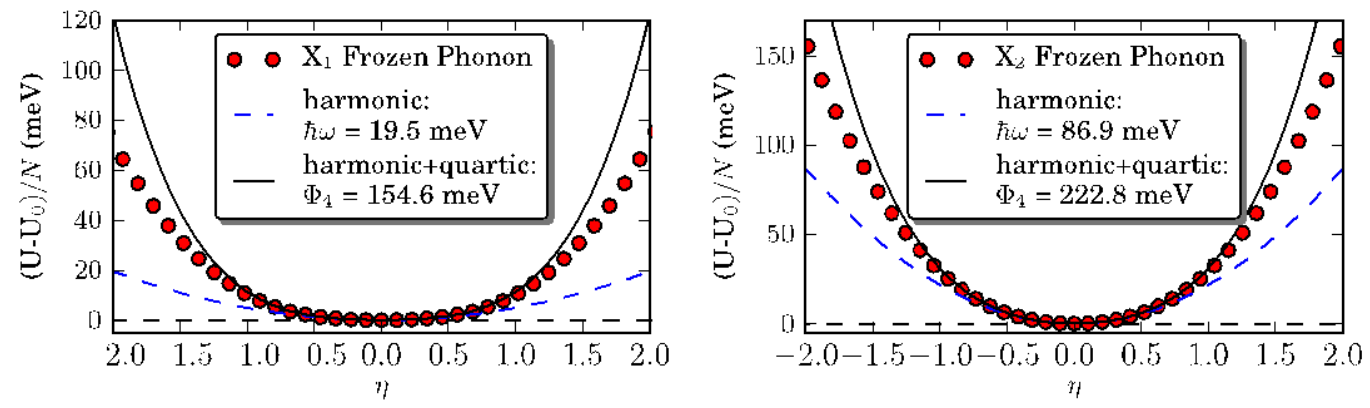

Fig.2. Frozen phonon total-energy calculations for the modes at $\mathrm{X}$ with frequencies $\sim 20 \mathrm{meV}$ (X1) and $\sim 85$ meV (X2), as a function of a displacement parameter $\eta$. Reproduced from Physical Review B (43).

Recently, Erea et al. (37) have performed ab-initio calculations of $\mathrm{T}_{\mathrm{c}}$ in PdH using newly developed Stochastic Self-Consistent Harmonic approximation (SSCHA) - see also Ref. (45). This work was followed by two papers demonstrating the effect of anharmonicity on calculated $\mathrm{T}_{\mathrm{c}}$ in $\mathrm{PtH}$ and $\mathrm{PdH}$ (45), and in other hydrides and chalcogenides (46). Their results show that the harmonic approximation describing the vibrations of atoms in solids can completely break down when the displacements of the atoms exceed the range in which the harmonic potential is valid. Such situations occur mostly for very light atoms like hydrogen, or in very soft effective potentials (47). In Fig.2 we reproduce the electron-phonon coupling calculation for PtH from Erea et al. (45). 


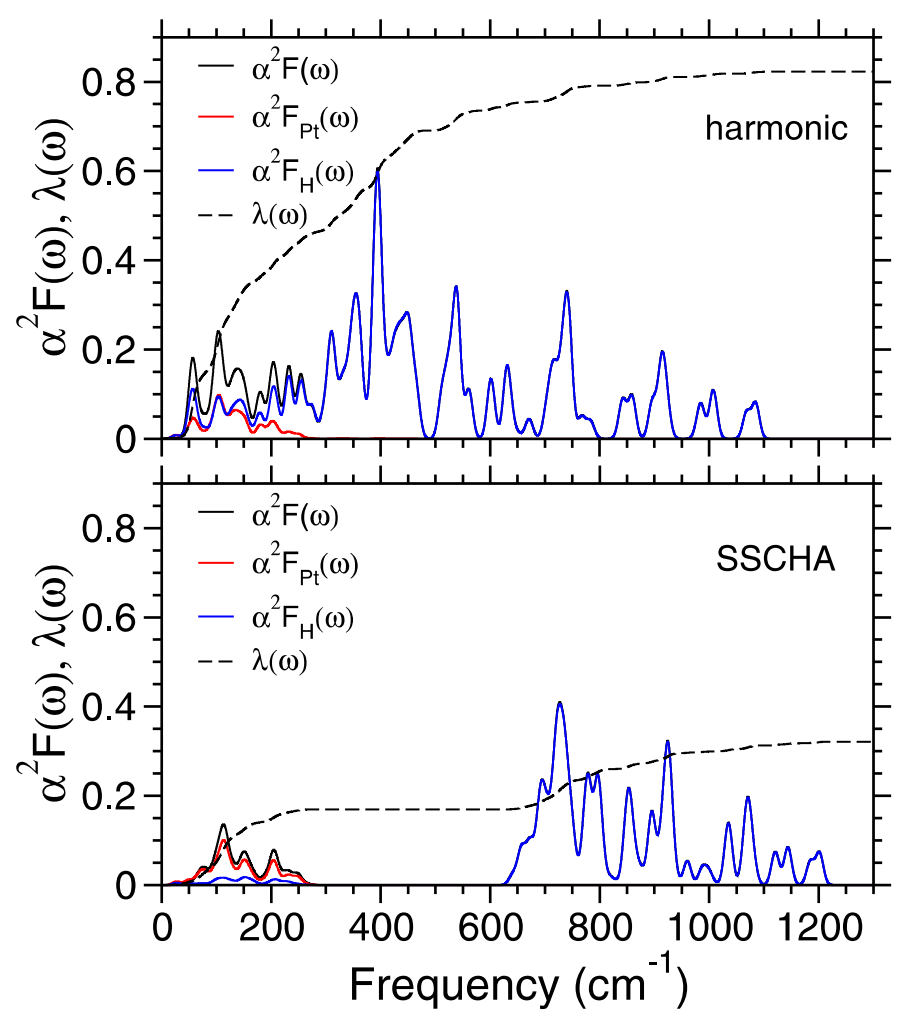

Fig.3. $\alpha^{2} \mathrm{~F}(\omega)$ and $\lambda(\omega)$ of PtH at $100 \mathrm{GPa}$ and $0 \mathrm{~K}$ in the harmonic approximation (top) and in the SSCHA (bottom). The contribution of Pt and $\mathrm{H}$ atoms to $\alpha^{2} \mathrm{~F}(\omega)$ is also shown. Reproduced from Phys. Rev. B., Erea et. al. (45).

As is clear from Fig. 2, the calculations in SSCHA formalism, taking account of anharmonic effects, reduce significantly theoretical estimation of electron-phonon coupling contribution due to vibrations of hydrogen atoms. The calculated anharmonic hydrogen vibrations are located much higher in frequency, than harmonic approximation would predict. According to Fig. 1, the contribution to the $T_{c}$ should dramatically decrease, since the phonon density of states is shifted by more than factor of 10 above the energy corresponding to $\mathrm{T}_{\mathrm{c}}$. 


\section{Hydrogen "doped" with other elements}

\section{A. Silane and its siblings}

The possibility that compressed hydrogen may convert to a metallic state under compression was first suggested in 1935 (48), but experimental realization of metallic hydrogen in its solid atomic form (49) remains a challenging goal. In 2004, Ashcroft suggested that dense hydrides of group IVa elements are promising candidates for realizing metallization of solid hydrogen at pressures considerably lower than may be necessary for solid hydrogen because hydrogen has already undergone a form of "chemical pre-compression" (6). The idea of diluting hydrogen with other elements is not new (50), but only recently experimental x-ray, Raman, transport, and infrared measurements as well as theoretical calculations have been performed on the group IVa hydrides (mostly silane $\mathrm{SiH}_{4}$ and germane $\mathrm{GeH}_{4}$ ) in order to explore their metallization and potential superconductivity suggested by $A \operatorname{shcroft}(6)$. Experimental (5l) and theoretical (52) results pointed to instability of germane at high pressures and even enabled measurements of high-quality $\mathrm{x}$-ray diffraction data on high-pressure germanium phases (51), which were possible due to perfect powder x-ray diffraction of germanium in a soft pressure medium $\left(\mathrm{H}_{2}\right)$ formed by decomposition of $\mathrm{GeH}_{4}$. 

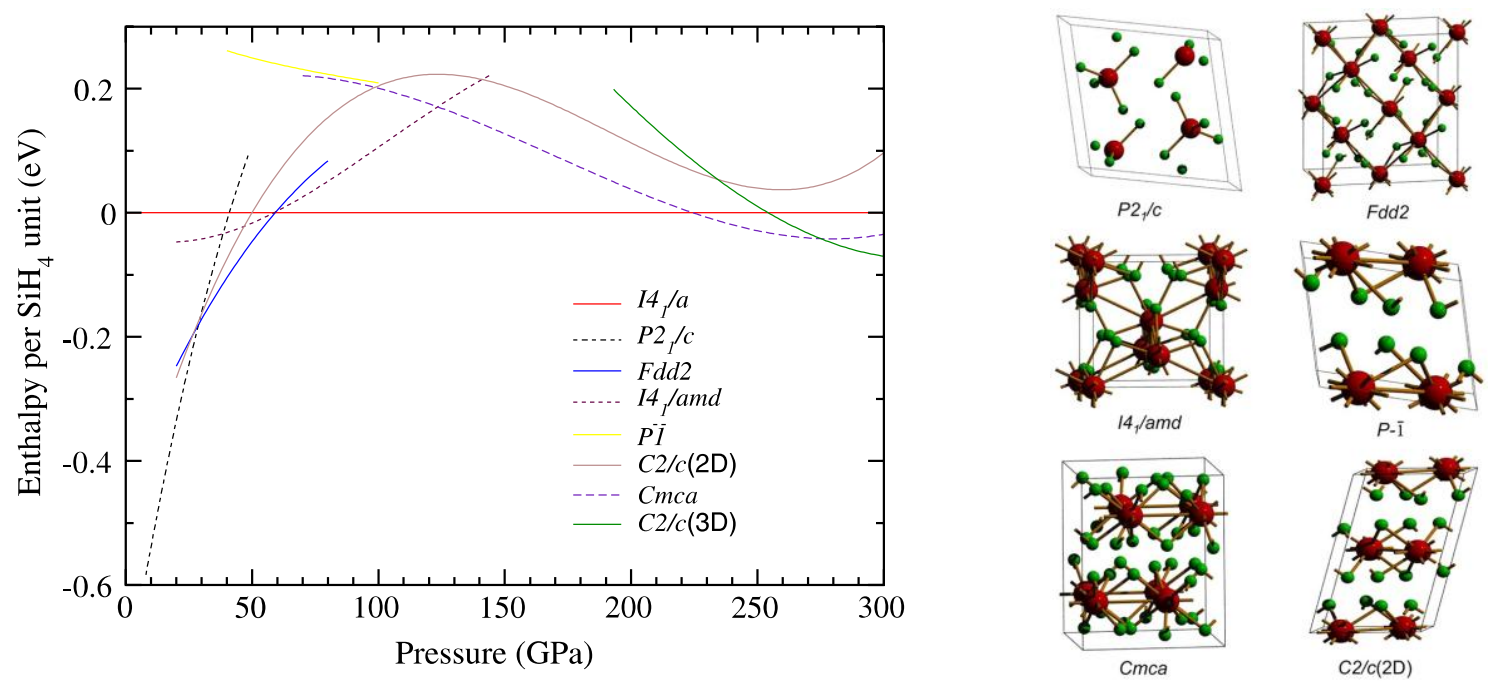

Fig.4. The enthalpy versus pressure for competitive structures of $\mathrm{SiH}_{4}$. The enthalpy of the $\mathrm{I}_{1} / \mathrm{a}$ phase is taken as the reference point. The "enthalpically" most favorable structures computed for SiH4 polymorphs at high pressures are shown on the right (53). The Cmca structure having a layered network is the most likely candidate of the metallic phase of $\mathrm{SiH}_{4}$ over a wide pressure range above $60 \mathrm{GPa}$. The superconducting transition temperature in this layered metallic phase is found to be in the range of $20-75 \mathrm{~K}$ (53).

The early theoretical work on silane (54-56) initiated significant experimental and theoretical efforts in search for superconductivity. Silane $\left(\mathrm{SiH}_{4}\right)$ has been the subject of several recent high-pressure studies. Chen et al. (57) used high-pressure spectroscopy to identify high-pressure behavior of silane. They found from Raman spectroscopy one fluid-solid transition at around 4.0 GPa and three solid-solid transitions near 6.5 GPa, 10 $\mathrm{GPa}$, and 26. $5 \mathrm{GPa}$ at room temperature. Four high- pressure solid phases were detected, which we named phase III, IV, V, and VI. The infrared results show an increase of the reflectivity starting at $60 \mathrm{GPa}(57)$, pointing at pressure-induced metallization, which confirms the prediction by Ashcroft (6). The results indicated that $\mathrm{SiH}_{4}$ is a good candidate for the realization of the metallization of hydrogen under pressure. Theoretical 
predictions by Chen et al. also favored high pressure superconductivity in silane (53) see Fig.4. The predictions were made by other groups (58-60), also indicating high pressure superconductivity in silane. Indeed, the superconductivity was found with $\mathrm{T}_{\mathrm{c}}$ approaching $20 \mathrm{~K}$ in transport measurements performed by Eremets et al. (18) - Fig.5. While silane remains stable below $50 \mathrm{GPa}(61)$, it was claimed that it decomposes above $50 \mathrm{GPa}(62)$ forming pure silicon $(\mathrm{Si})$ and hydrogen $\left(\mathrm{H}_{2}\right)$, which in turn reacts with the metallic gasket $(\mathrm{Re})$ or electrical leads $(\mathrm{Pt})$ and may be responsible for a superconductivity and $\mathrm{x}$-ray diffraction from platinum hydride observed in experiments by Eremets (18) and attributed to metallic silane. Further experiments by Hanfland et al. pointed to amorphisation of silane at $60 \mathrm{GPa}$ and to formation of polymeric silane around $100 \mathrm{GPa}(63)$. This study also found that silicon and hydrogen react above $100 \mathrm{GPa}$ to form stable polymeric phases of silane (63). In the absence of decomposition, silane was found to remain partially transparent and nonmetallic to at least $150 \mathrm{GPa}$ with a band gap constrained between 0.6 and $1.8 \mathrm{eV}(64)$.
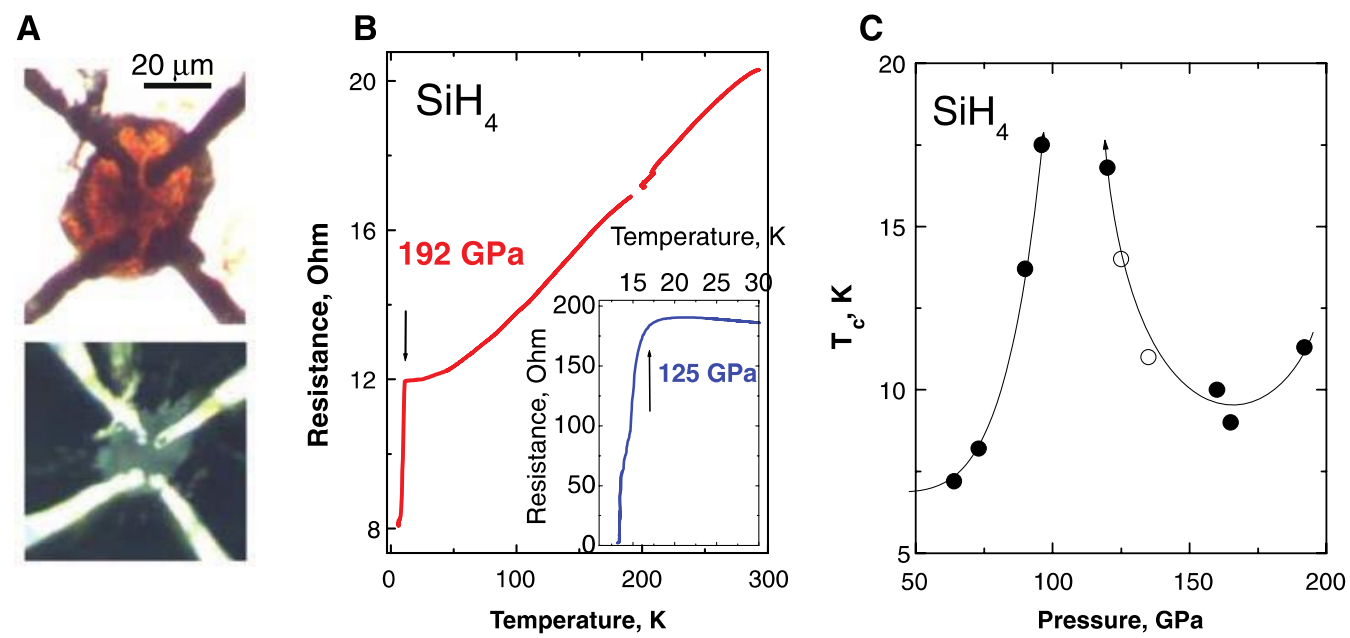

Fig.5. Electrical resistance and superconductivity in silane. (A) Photo- graphs of silane at $125 \mathrm{GPa}$ taken in transmitted (above) and reflected (be- low) light after annealing at $\mathrm{T}=400^{\circ} \mathrm{C}$. Silane is transparent at this pressure; nevertheless, it noticeably reflects light. (B) A representative superconducting step on the 
temperature dependence of resistance of silane at $192 \mathrm{GPa}$ and $125 \mathrm{GPa}$ (inset). Arrows indicate Tc. Electri- cal measurements were performed be- fore the annealing, when an essential part of the sample remained in the metallic phase. (C) Pressure depen- dence of the critical transition temper- ature Tc of silane. Reproduced from Science (18).
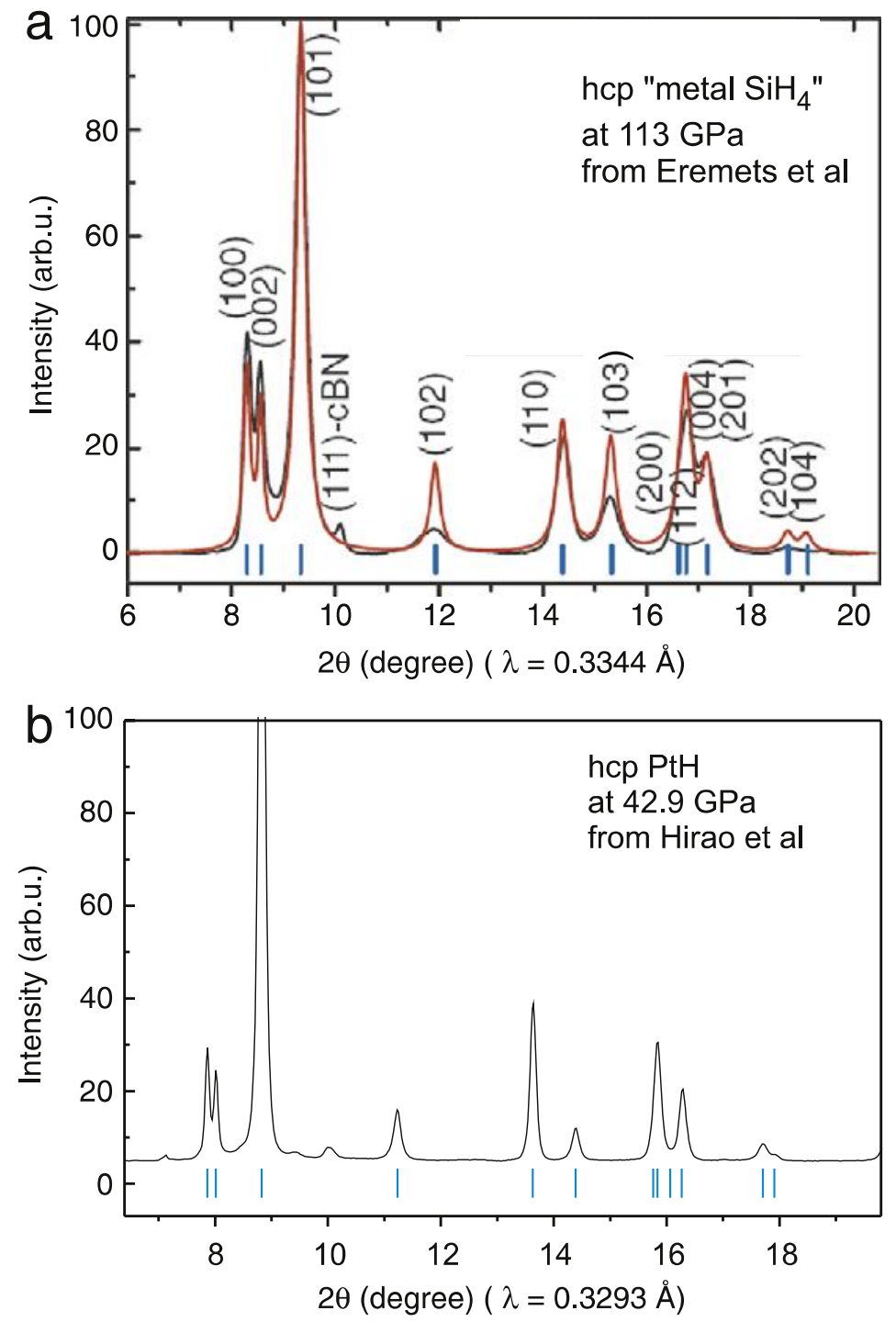

Fig.6. $\mathrm{SiH}_{4}-\mathrm{PtH}$ controversy. Powder diffraction spectra of (a) the claimed "hcp phase of metallic silane', (18) and (b) hcp phase of PtH synthesized at high pressure. The tick marks in (b) show peak positions calculated from the reported lattice parameters for $\mathrm{PtH}$ in its hcp phase: $\mathrm{a}=2.773(1) \mathrm{A} \square$, and $\mathrm{c}=4.713$ (1) A $\square$. reproduced from Solid State Cummunications, Degtyareva et al. (62). 
Experimentally, controversies regarding the decomposition of silane at high compression and its superconductivity are not resolved yet - Fig.6. We have performed both resistivity and magnetic susceptibility experiments (65) in platinum hydride formed in situ at high pressure to check the suggested effect (62) of electrical leads in previous experiments on silane (18). We were not able to confirm superconductivity of platinum hydride up to $100 \mathrm{GPa}$. Further magnetic susceptibility experiments may be required with silane loaded in a gold-lined and cBN gasket to exclude the effect of a metallic gasket and to contain possibly released hydrogen. In view of the theoretical results on anharmonic suppression of $\mathrm{T}_{\mathrm{c}}$ in $\mathrm{PtH}$, the high $\mathrm{T}_{\mathrm{c}}$ observed in (18) may belong to superconducting phases of $\mathrm{SiH}_{\mathrm{x}}$ compounds with different stoichiometry (66-69) or to polymeric phases of silane (63).

Stannane $\left(\mathrm{SnH}_{4}\right)(60)$ and plumbane $\left(\mathrm{PbH}_{4}\right)(70)$ were proposed as high-pressure (high-temperature) superconductor $\left(\mathrm{SnH}_{4}\right)$ and liquid metal $\left(\mathrm{PbH}_{4}\right)$, but experimental attempts to create these compounds at high pressure have not been reported so far.

\section{B. Polyhydrides of alkali and alkaline earth metals}

The content of this section will cover exclusively recent theoretical developments, which are very new and mostly did not find experimental confirmation at his moment. However, experiments are underway and experimentalists would benefit from a concise review of predicted polyhydrides, which have unusual compositions and defy our intuitive expectations. 
As a very first development in that direction, metallic polyhydrides of lithium have been proposed $(19,20)$ to exist at pressures above $100 \mathrm{GPa}$, well below the expected metallization pressure of pure hydrogen (21). Stable polyhydrides of other alkali metals have been predicted recently by theoretical analysis of $\mathrm{MHn}(\mathrm{M}=\mathrm{Li}, \mathrm{Na}, \mathrm{K}, \mathrm{Rb}, \mathrm{Cs})$ compounds with variable hydrogen composition $(19,20,22-25)$. The compounds with $\mathrm{n} \geq 2$ are expected to become stable at pressures as low as few GPa in the case of Cs and above $100 \mathrm{GPa}$ in the case of $\mathrm{Li}$.

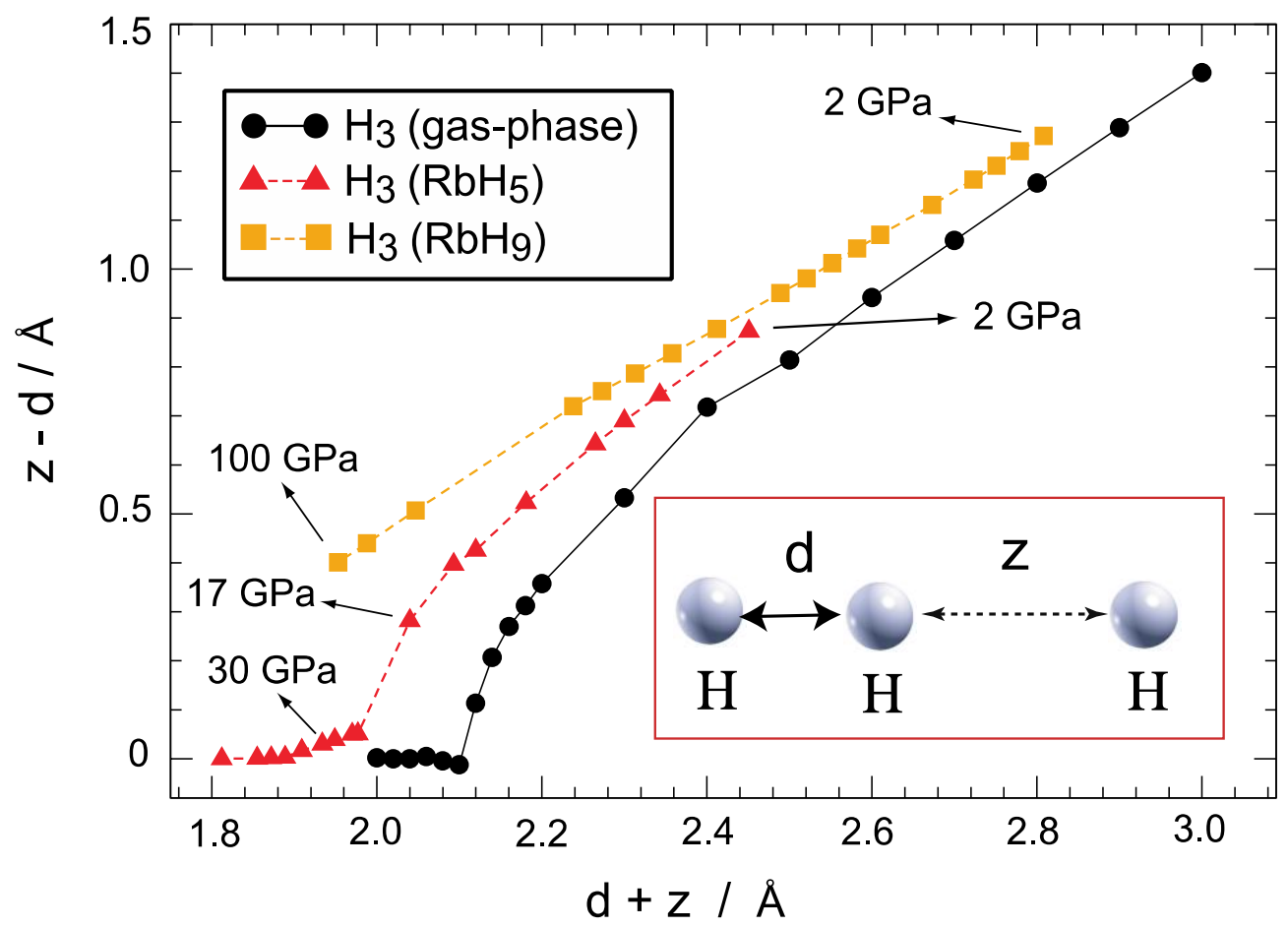

Fig. 7. The difference between the $\mathrm{z}$ and d distances in an $\mathrm{H}-\cdots \mathrm{H} 2$ fragment (see inset) is plotted vs. their sum for an isolated $\mathrm{H}_{3}-$ molecule in the gas phase. During the optimization, the total length of the molecule was constrained and the central atom was allowed to relax freely. These values are also illustrated for an $\mathrm{H}^{-} \cdots \mathrm{H} 2$ unit in $\mathrm{lp}-\mathrm{RbH} 9$ and $\mathrm{lp}-\mathrm{RbH} 5$. Reproduced from Chem. Eur. J., Hooper et al. $(71)$. 
New polyhydride phases are also anticipated for alkaline earth metals $(24,72,73)$. The theoretically predicted polyhydrides are not only stabilized by compression, but are expected to metalize and exhibit superconducting properties at lower pressures than the constituent parts--the monohydride and hydrogen. Critical superconducting temperatures as high as $235 \mathrm{~K}$ have been predicted for polyhydrides of $\mathrm{Ca}(72)$.

These compounds provide new chemical means to pre-compress hydrogen molecules and to facilitate the creation of metallic superconducting hydrogen (5) with record high critical superconducting temperatures. Notably, linear $\mathrm{H}_{3}$ - ions were predicted to form in polyhydrides of softer alkali metals, for example in $\mathrm{RbH}_{5}(71)$ and $\mathrm{CsH}_{3}(25)$. Such $\mathrm{H}_{3}$ ions (see for example Fig. 7) have a tendency to be stabilized in a linear configuration, contrary to triangular-shaped $\mathrm{H}_{3}+$ ions $(74,75)$. Symmetric $\mathrm{H}_{3}$ - ions with interatomic distance $1.06 \AA$ and with the lowest potential barrier (76) were discussed as transition states in hydrogen exchange processes of metal complexes (77). $\mathrm{H}_{3}$ - and $\mathrm{D}_{3}$ - ions were experimentally observed in discharge plasmas only recently (78). The chains of $\mathrm{H}_{3}$ - ions that were found theoretically in $\mathrm{RbH}_{5}(25)$ and $\mathrm{CsH}_{3}(71)$, resemble one-dimensional hydrogen chains of ambient pressure metastable metallic hydrogen phases predicted in 1976 by Browman and Kagan (17). Such chains were also extensively discussed as a simplest model of strong correlations in a linear chain of hydrogen atoms $(79,80)$. In recent paper, Grabowski and Hoffman (81) have considered stabilization effect of the excited state of $\mathrm{H}_{3}$ - anion with hydrogen separation $0.93 \AA$ on a (LiHHHLi)+ pentaatomic cation and also found (81) a linear coordinated arrangement. Other local stable clusters have been proposed. The pure hydrogen clusters $\left(\mathrm{H}_{3}+, \mathrm{H}_{3^{-}}\right.$, etc...) and 
clusters of hydrogen with other elements (81) may serve as building blocks of pressureinduced metallic phases. We are only starting to develop our intuitive understanding of emerging new strongly compressed materials with unexpected chemistry and physical properties.

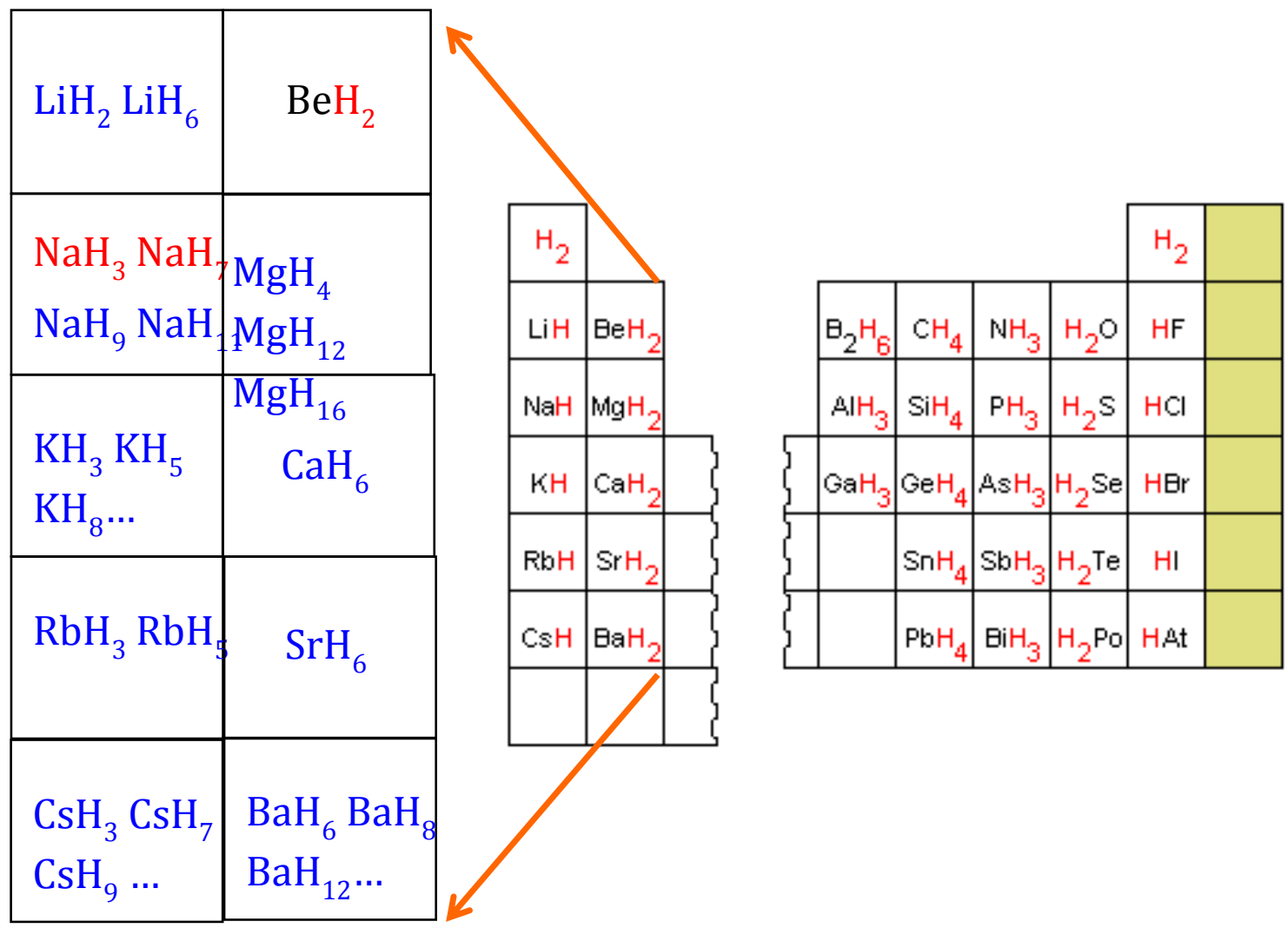

Fig.8. The modified Periodic Table shows hydrides of the elements belonging to main groups. A separate block on the left summarizes a variety of polyhydrides predicted recently and discussed in this section. $\mathrm{NaH}_{3}$ and $\mathrm{NaH}_{7}$ were synthesized recently in our group (submitted).

Alkali and alkaline earth polyhydrides predicted recently are summarized in Fig. 8. Apparently, polyhydrides of other main group elements may exist, as was reported 
recently, for example, in Xe (82). Group VII polyhydrides, if they exist, would be "hole doped", as compared to alkali and alkaline earth, which are "electron doped". We may expect very different properties for hole doped polyhydrides, and also quite different local arrangements, possibly based on triangular $\mathrm{H}_{3}+$ cation (75). In this review we did not discuss polyhydrides of transition metals or rare earth metals at high pressure, which have only limited impact in current literature (83) and have limited connection to highpressure experiments at this time, with only one candidate for "non-interstitial hydride", $\mathrm{IrH}_{3}$ (84). Existing classical "interstitial" metal hydrides were briefly discussed in section 1 .

As a concluding remark, despite a wealth of theoretically predicted high pressure polyhydride structures, none of the predictions have been confirmed experimentally until now in the published literature. Also, the claims of metallic and superconducting phases should be considered with caution, and further in-depth theoretical analysis of superconducting properties may be required.

We have tried to create polyhydrides of Li (without success, see also Ref. (85)) and $\mathrm{Na}\left(\mathrm{NaH}_{3}\right.$ and $\mathrm{NaH}_{7}$ synthesized, work in progress) (86). We are optimistic and are looking forward to new polyhydrides coming to life very soon.

Notes added. When this paper was in review, the paper by Drozdov et. al. was published reporting nearly $190 \mathrm{~K}$ conventional superconductivity in $\mathrm{H}_{2} \mathrm{~S}$ hydride at 200 GPa (87). The experimental procedure and theoretical results by Duan et. al. (88) suggest that observed high $\mathrm{T}_{\mathrm{c}}$ values may correspond to a polyhydride $\mathrm{H}_{3} \mathrm{~S}$ phase forming at high pressures when the sample is warmed up to room temperature (87). The experiments have reported superconductivity from resistivity measurements. If these results will be 
confirmed by magnetic susceptibility measurements, there will remain no doubt that conventional phonon driven conductivity is possible close to room temperature. It is interesting to note that hydrogen in $\mathrm{H}_{3} \mathrm{~S}$ and its homologues would represent a test case to study a long sought "hole doped" metallic hydrogen, as discussed in this section.

\section{Prospects for room temperature superconductor based on hydrogen- rich materials: concluding remarks.}

Current candidate materials for high-temperature or even room-temperature superconductivity were discussed at length in previous sections. However, none of the predicted materials matches pure hydrogen as a candidate for room temperature superconductivity - Fig. 9. Pure hydrogen remains in the focus of current research, and quite remarkable progress in bringing hydrogen to nearly $400 \mathrm{GPa}$ has been reported recently (89-94). 


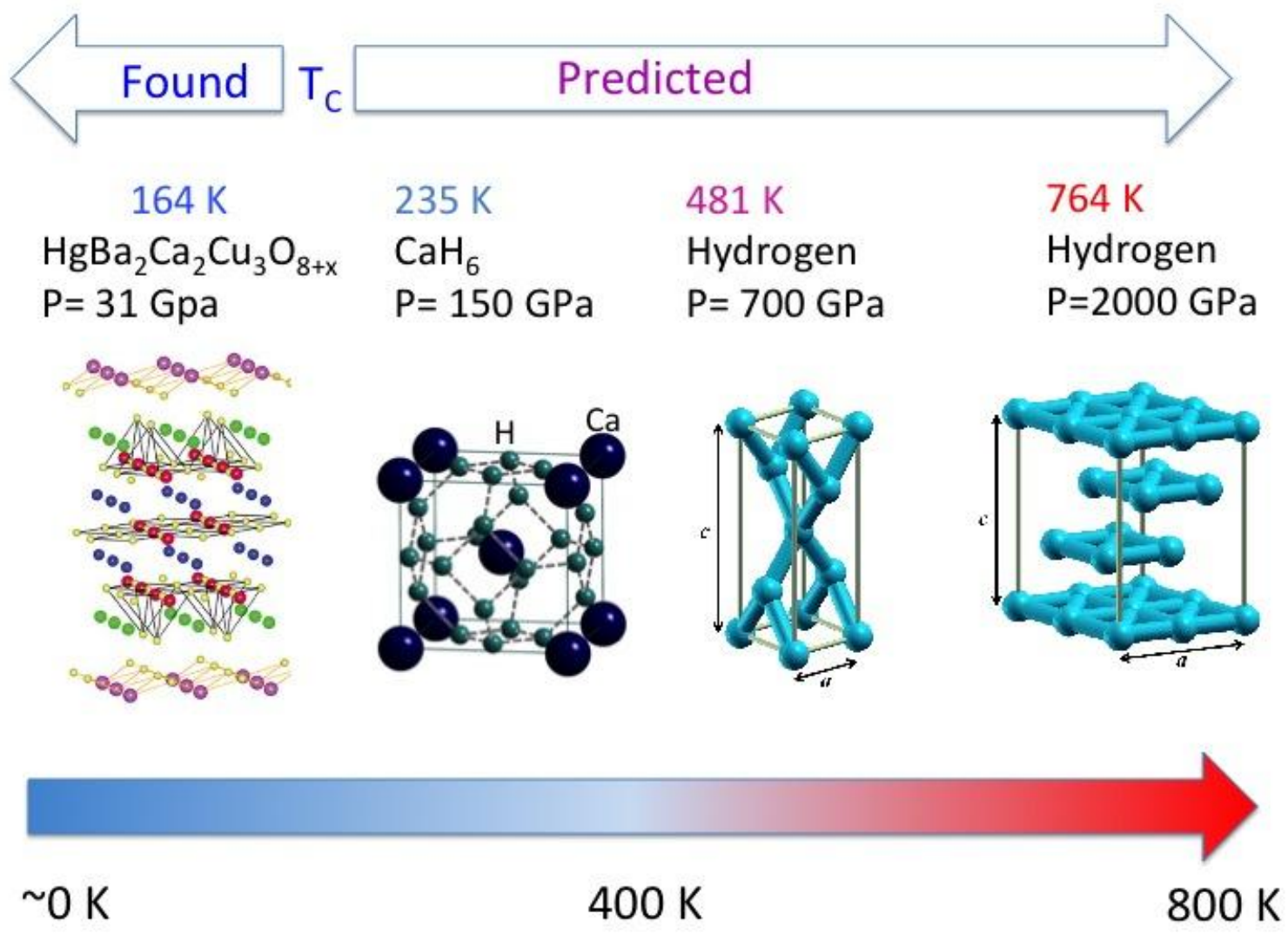

Fig.9. The highest $\mathrm{T}_{\mathrm{c}}$ reached so far in a mercury based cuprate is $164 \mathrm{~K}$ at $30 \mathrm{GPa}(95)$. $\mathrm{T}_{\mathrm{c}}$ 's close to room temperature were predicted for $\mathrm{CaF}_{6}$ at $150 \mathrm{GPa}$, and $\mathrm{T}_{\mathrm{c}}$ 's well above room temperature are expected in metallic hydrogen (atomic form) (49). Recent claim of superconducting hydrogen sulfide (87) may change the record of the $\mathrm{T}_{\mathrm{c}}$ to $190 \mathrm{~K}$, if confirmed.

Conducting hydrogen has been claimed be Eremets and Troyan (96), however, this work has received a serious criticism (97). If this experiment will stand the test of time, it has reported semimetalic, poorly conducting molecular phase of hydrogen (97). It should be noted here that high Tc's were also predicted in metallic molecular forms of hydrogen - see $(98,99)$ and references therein.

Apparently, room temperature superconducting atomic hydrogen (49) is still on the radar for experimentalists. Experimental challenges are significant, but the task of 
reaching the immense pressures required to metalize hydrogen may be facilitated by recently developed double-stage diamond anvil technique (100). A combination of such technique with precise photolithography or Focused Ion Beam (FIB) techniques (101) may help to manufacture tiny electrical leads in a double-stage diamond anvil cell (DAC) and to probe metallization of hydrogen above $400 \mathrm{GPa}$.

New predicted polyhydrides (section 3) also hold a great promise to have high critical superconducting temperatures. However, predicted high $\mathrm{T}_{\mathrm{c}}$ values should be carefully checked both by experiment and by more accurate calculations taking account of anharmonic effects. In view of recently reported superconductivity with Tc $190 \mathrm{~K}$ in hydrogen sulfide (87), the "hole doped" hydrogen in combination with chalcogens or pnictides, or both, is a promising chemical route to new high- $\mathrm{T}_{\mathrm{c}}$ superconducting materials.

\section{Appendix: Properties of the superconducting state of hydrides}

Table 1. The properties of hydride materials available in the literature, such as critical fields $\left(\mathrm{H}_{\mathrm{c} 1}\right.$ and $\left.\mathrm{H}_{\mathrm{c} 2}\right)$, pressure effect on the $\mathrm{T}_{\mathrm{c}}$, superconducting gap, isotope effects, and other available information, are listed along with the references to original publications. The information available for metal hydrides is limited due to difficulties in growing stoichiometric samples. This may also explain a large scatter in experimentally measured parameters. The information for $\mathrm{PdH}(\mathrm{D})$ and $\mathrm{ThH}(\mathrm{D})$ is dominant due to the large interest to the inverse isotope effect in these hydrides and their enhanced $\mathrm{T}_{\mathrm{c}}$ values.

It should be also noted that hydrides of many transition and rare earth metals have been studied but no superconductivity has been found in them - see e. g. Refs. (30), (102) 
for a list of such hydrides. Many examples are also given (30) showing that small amounts of hydrogen dissolved in a superconducting metal usually suppress its $T_{c}$.

\begin{tabular}{|c|c|c|c|}
\hline Hydride & $\mathbf{T}_{\mathbf{c}}(\mathbf{K})$ & Properties of the superconducting state & Reference \\
\hline $\begin{array}{l}\mathrm{H}_{2} \mathrm{~S} \\
\mathrm{D}_{2} \mathrm{~S} \\
\mathrm{H}_{\mathrm{x}} \mathrm{S} \\
\mathrm{D}_{\mathrm{x}} \mathrm{S} \\
\mathrm{H}_{2} \mathrm{~S} \\
\mathrm{H}_{\mathrm{x}} \mathrm{S}\end{array}$ & $\begin{array}{l}30-150 \\
20-50 \\
160-190 \\
90 \\
60 \\
185\end{array}$ & $\begin{array}{l}\mathrm{P} \text { from } 110 \mathrm{GPa} \text { to } 200 \mathrm{GPa} \\
\mathrm{P} \text { from } 100 \mathrm{GPa} \text { to } 175 \mathrm{GPa} \\
\mathrm{P} \text { from } 150 \text { to } 200 \mathrm{GPa} \text {, possible } \mathrm{x}=3 \\
\mathrm{P}=170 \mathrm{GPa} \\
H_{c 2}=25 \mathrm{~T} \\
H_{c 2}=72 \mathrm{~T}\end{array}$ & $\begin{array}{l}(87),(103), \\
(88)\end{array}$ \\
\hline $\mathrm{SiH}_{4}$ & 17.5 & at $\mathrm{P} \sim 100 \mathrm{GPa}$ & (18) \\
\hline $\mathrm{PdH}_{0.97}$ & 8.3 & $H_{c 2}=1.7 \mathrm{~T}$ & $(104)$ \\
\hline $\mathrm{PdH}_{0.97}$ & 6.6 & $H_{c 2}=0.18 \mathrm{~T}$ & $(105)$ \\
\hline $\mathrm{PdH}_{0.996}$ & 10.4 & $H_{c 2}=0.09 \mathrm{~T}$ & $(106)$ \\
\hline $\mathrm{PdH}_{\mathrm{x}}$ & $\begin{array}{l}3.4 \\
3.6 \\
4.2 \\
5.5 \\
6.2 \\
7.7\end{array}$ & $\begin{array}{lcc}H_{c 2}(\mathrm{~T}) & d H_{c 2} / d T(\mathrm{~T} / \mathrm{K}) & \rho_{n}\left(10^{-5} \Omega \mathrm{cm}\right) \\
0.219 & 0.0645 & 1.23 \\
0.238 & 0.0660 & 1.27 \\
0.252 & 0.0600 & 1.04 \\
0.250 & 0.0455 & 0.85 \\
0.218 & 0.0350 & 0.61 \\
0.243 & 0.0315 & 0.60 \\
\rho_{\mathrm{n}}-\text { residual resistance } & \end{array}$ & (107) \\
\hline $\mathrm{PdD}_{0.9}$ & 5.5 & $2 \Delta_{0} / k_{B} T_{c}=3.6$ & (108) \\
\hline $\mathrm{PdH}_{\mathrm{x}}$ & 4.6 & $2 \Delta_{0} / k_{B} T_{c}=3.8$ & $(109)$ \\
\hline $\mathrm{PdH}_{\mathrm{x}}$ & 4.6 & $2 \Delta_{0} / k_{B} T_{c}=2.6$ & $(110)$ \\
\hline $\begin{array}{l}\mathrm{PdH} \\
\mathrm{PdD}\end{array}$ & $\begin{array}{l}8.8 \\
11.05\end{array}$ & $\begin{array}{l}d \ln T_{d} d d P=-6.8 * 10^{-3} \mathrm{kbar}^{-1} \\
d \ln T_{d} d d P=-5.0^{*} 10^{-3} \mathrm{kbar}^{-1}\end{array}$ & $(111)$ \\
\hline $\begin{array}{l}\mathrm{Th}_{4} \mathrm{H}_{15} \\
\mathrm{Th}_{4} \mathrm{D}_{15}\end{array}$ & $\begin{array}{l}8.3 \\
8.3\end{array}$ & $\begin{array}{l}H_{c 2}=2.5-3.0 \mathrm{~T} \\
H_{c 2}=2.5-3.0 \mathrm{~T}\end{array}$ & $(31)$ \\
\hline $\mathrm{Th}_{4} \mathrm{H}_{15}$ & 7.62 & $d \ln T_{c} / d P=5.5^{*} 10^{-3} \mathrm{kbar}^{-1}$ & $(112)$ \\
\hline $\begin{array}{l}\mathrm{Th}_{4} \mathrm{H}_{15} \\
\mathrm{Th}_{4} \mathrm{D}_{15}\end{array}$ & $\begin{array}{l}7.9 \\
7.9\end{array}$ & $\begin{array}{l}2 \Delta_{0} / k_{B} T_{c}=3.42 \\
2 \Delta_{0} / k_{B} T_{c}=3.47\end{array}$ & (113) \\
\hline $\begin{array}{l}\mathrm{Th}_{4} \mathrm{H}_{15} \\
\mathrm{Th}_{4} \mathrm{D}_{15}\end{array}$ & $\begin{array}{l}7.97 \\
8.00\end{array}$ & $\begin{array}{l}\Theta_{D}=211.5 \mathrm{~K}, \gamma=8.07 \mathrm{~mJ} * \mathrm{~K}^{-2} / \text { mole Th } \\
\Theta_{D}=216.5 \mathrm{~K}, \gamma=7.84 \mathrm{~mJ} * \mathrm{~K}^{-2} / \text { mole Th }\end{array}$ & $(113)$ \\
\hline $\begin{array}{l}\mathrm{MoH}_{1.27} \\
\mathrm{MoD}_{1.26}\end{array}$ & $\begin{array}{l}0.92 \\
1.4\end{array}$ & $\begin{array}{l}H_{c}=60 \mathrm{mT} \\
H_{c}=30 \mathrm{mT}\end{array}$ & (114) \\
\hline
\end{tabular}




\section{Acknowledgements}

This work was supported by Carnegie Institution for Science, and DOE BES under contracts DE-FG02-02ER45955 and DE-FG02-99ER45775. The author benefited from numerous discussions with W. Grochala, and D. Y. Kim, and M. Somayazulu. 


\section{References}

1. A. Janotti, C. G. Van de Walle, Hydrogen multicentre bonds. Nat Mater 6, 44-47 (2007); published online Epub01//print (http://www.nature.com/nmat/journal/v6/n1/suppinfo/nmat1795_S1.html).

2. S. Hammes-Schiffer, Comparison of Hydride, Hydrogen Atom, and ProtonCoupled Electron Transfer Reactions. ChemPhysChem 3, 33-42 (2002)10.1002/1439-7641(20020118)3:1<33::AID-CPHC33>3.0.CO;2-6).

3. R. G. Pearson, The transition-metal-hydrogen bond. Chemical Reviews 85, 41-49 (1985); published online Epub1985/02/01 (10.1021/cr00065a002).

4. J. Bardeen, L. N. Cooper, J. R. Schrieffer, Theory of Superconductivity. Phys. Rev. 108, 1175-1204 (1957).

5. N. W. Ashcroft, Metallic Hydrogen: A High-Temperature Superconductor? Physical Review Letters 21, 1748-1749 (1968).

6. N. W. Ashcroft, Hydrogen Dominant Metallic Alloys: High Temperature Superconductors? Phys. Rev. Lett. 92, 187002 (2004).

7. B. Stritzker, High superconducting transition temperatures in the palladium-noble metal-hydrogen system. Z. Physik 268, 261-264 (1974); published online Epub1974/06/01 (10.1007/BF01669889).

8. A. Leiberich, W. Scholz, W. J. Standish, C. G. Homan, Superconductivity in Hcharged Cu-implanted Pd. Physics Letters A 87, 57-60 (1981); published online Epub12/21/ (http://dx.doi.org/10.1016/0375-9601(81)90615-0).

9. T. Skoskiewicz, A. W. Szafranski, W. Bujnowski, B. Baranowski, Isotope effect in the superconducting palladium-hydrogen-deuterium system. Journal of Physics C: Solid State Physics 7, 2670 (1974).

10. P. Tripodi, D. Di Gioacchino, R. Borelli, J. D. Vinko, Possibility of high temperature superconducting phases in $\mathrm{PdH}$. Physica C: Superconductivity 388389, 571-572 (2003); published online Epub5// (http://dx.doi.org/10.1016/S09214534(02)02745-4).

11. W. Grochala, P. P. Edwards, Thermal Decomposition of the Non-Interstitial Hydrides for the Storage and Production of Hydrogen. Chemical Reviews 104, 1283-1316 (2004); published online Epub2004/03/01 (10.1021/cr030691s).

12. V. V. Struzhkin, B. Militzer, W. L. Mao, H.-k. Mao, R. J. Hemley, Hydrogen Storage in Molecular Clathrates. Chemical Reviews 107, 4133-4151 (2007); published online Epub2007/10/01 (10.1021/cr050183d).

13. J. Goldsmith, A. G. Wong-Foy, M. J. Cafarella, D. J. Siegel, Theoretical Limits of Hydrogen Storage in Metal-Organic Frameworks: Opportunities and Trade-Offs. Chemistry of Materials 25, 3373-3382 (2013); published online Epub2013/08/27 (10.1021/cm401978e).

14. Z. Yang, Y. Xia, R. Mokaya, Enhanced Hydrogen Storage Capacity of High Surface Area Zeolite-like Carbon Materials. Journal of the American Chemical Society 129, 1673-1679 (2007); published online Epub2007/02/01 $(10.1021 / \mathrm{ja} 067149 \mathrm{~g})$. 
15. J. Yang, A. Sudik, C. Wolverton, D. J. Siegel, High capacity hydrogen storage materials: attributes for automotive applications and techniques for materials discovery. Chemical Society Reviews 39, 656-675 (2010)10.1039/B802882F).

16. E. Babaev, A. Sudbø, N. W. Ashcroft, A superconductor to superfluid phase transition in liquid metallic hydrogen. Nature 431, 666-668 (2004).

17. E. G. Brovman, Y. Kagan, A. Kholas, Structure of Metalic Hydrogen at Zero Pressure. Sov. Phys. JETP 34, 1300-1315 (1972).

18. M. I. Eremets, I. A. Trojan, S. A. Medvedev, J. S. Tse, Y. Yao, Superconductivity in Hydrogen Dominant Materials: Silane. Science 319, 1506-1509 (2008); published online EpubMarch 14, 2008 (10.1126/science.1153282).

19. E. Zurek, R. Hoffmann, N. W. Ashcroft, A. R. Oganov, A. O. Lyakhov, A little bit of lithium does a lot for hydrogen. Proceedings of the National Academy of Sciences 106, 17640-17643 (2009); published online EpubOctober 20, 2009 (10.1073/pnas.0908262106).

20. J. P. Chris, R. J. Needs, Ab initio random structure searching. Journal of Physics: Condensed Matter 23, 053201 (2011).

21. J. M. McMahon, D. M. Ceperley, Ground-State Structures of Atomic Metallic Hydrogen. Physical Review Letters 106, 165302 (2011).

22. P. Baettig, E. Zurek, Pressure-Stabilized Sodium Polyhydrides: NaH_\{n\} (n\&gt;1). Physical Review Letters 106, 237002 (2011).

23. J. Hooper, E. Zurek, High Pressure Potassium Polyhydrides: A Chemical Perspective. The Journal of Physical Chemistry C 116, 13322-13328 (2012); published online Epub2012/06/21 (10.1021/jp303024h).

24. J. Hooper, B. Altintas, A. Shamp, E. Zurek, Polyhydrides of the Alkaline Earth Metals: A Look at the Extremes under Pressure. The Journal of Physical Chemistry C 117, 2982-2992 (2013); published online Epub2013/02/14 (10.1021/jp311571n).

25. A. Shamp, J. Hooper, E. Zurek, Compressed Cesium Polyhydrides: Cs+ Sublattices and H3- Three-Connected Nets. Inorganic Chemistry 51, 9333-9342 (2012); published online Epub2012/09/03 (10.1021/ic301045v).

26. W. L. McMillan, Transition temperature of strong-coupled superconductors. Phys. Rev. 167, 331-344 (1968).

27. P. B. Allen, R. C. Dynes, Transition temperature of strong-coupled superconductors reanalyzed. Physical Review B 12, 905-922 (1975); published online Epub08/01/ (

28. P. Morel, P. W. Anderson, Calculation of the Superconducting State Parameters with Retarded Electron-Phonon Interaction. Physical Review 125, 1263-1271 (1962); published online Epub02/15/ (

29. B. Mitrović, J. P. Carbotte, Functional derivative $\delta \mathrm{Tc} / \delta \alpha 2(\Omega) \mathrm{F}(\Omega)$ for weak coupling superconductors. Solid State Communications 37, 1009-1013 (1981); published online Epub3// (http://dx.doi.org/10.1016/0038-1098(81)91206-0).

30. B. Stritzker, H. Wühl, in Hydrogen in Metals II, G. Alefeld, J. Völkl, Eds. (Springer Berlin Heidelberg, 1978), vol. 29, chap. 6, pp. 243-272.

31. C. B. Satterthwaite, I. L. Toepke, Superconductivity of Hydrides and Deuterides of Thorium. Physical Review Letters 25, 741-743 (1970); published online Epub09/14/ ( 
32. T. Skoskiewicz, Superconductivity in the palladium-hydrogen and palladiumnickel-hydrogen systems. physica status solidi (a) 11, K123-K126 (1972)10.1002/pssa.2210110253).

33. B. N. Ganguly, Anharmonicity and superconductivity in metal hydrides. I. Formulation. Physical Review B 14, 3848-3852 (1976).

34. B. M. Klein, E. N. Economou, D. A. Papaconstantopoulos, Inverse Isotope Effect and the $\mathrm{x}$ Dependence of the Superconducting Transition Temperature in $\mathrm{PdHx}$ and PdDx. Physical Review Letters 39, 574-577 (1977); published online Epub08/29/ (

35. J. P. Burger, Electron-phonon coupling and superconductivity in metal-hydrogen systems. Journal of the Less Common Metals 101, 53-67 (1984); published online Epub8// (http://dx.doi.org/10.1016/0022-5088(84)90087-0).

36. P. M. Laufer, D. A. Papaconstantopoulos, Theory of superconductivity in palladium $<\mathrm{i}>-</ \mathrm{i}>$ noble-metal hydrides. Physical Review B 33, 5134-5137 (1986); published online Epub04/01/ (

37. I. Errea, M. Calandra, F. Mauri, First-Principles Theory of Anharmonicity and the Inverse Isotope Effect in Superconducting Palladium-Hydride Compounds. Physical Review Letters 111, 177002 (2013); published online Epub10/25/ (

38. D. Y. Kim, R. H. Scheicher, H.-k. Mao, T. W. Kang, R. Ahuja, General trend for pressurized superconducting hydrogen-dense materials. Proceedings of the National Academy of Sciences 107, 2793-2796 (2010); published online EpubFebruary 16, 2010 (10.1073/pnas.0914462107).

39. D. Y. Kim, R. H. Scheicher, C. J. Pickard, R. J. Needs, R. Ahuja, Predicted Formation of Superconducting Platinum-Hydride Crystals under Pressure in the Presence of Molecular Hydrogen. Physical Review Letters 107, 117002 (2011).

40. A. Rahman, K. Sköld, C. Pelizzari, S. K. Sinha, H. Flotow, Phonon spectra of nonstoichiometric palladium hydrides. Physical Review B 14, 3630-3634 (1976); published online Epub10/15/ (

41. B. M. Klein, R. E. Cohen, Anharmonicity and the inverse isotope effect in the palladium-hydrogen system. Physical Review B 45, 12405-12414 (1992); published online Epub06/01/ (

42. J. P. Carbotte, F. Marsiglio, in The Physics of Superconductors, K. H. Bennemann, J. B. Ketterson, Eds. (Springer Berlin Heidelberg, 2003), chap. 4, pp. 233-345.

43. B. Rousseau, A. Bergara, Giant anharmonicity suppresses superconductivity in AlH_3 under pressure. Physical Review B 82, 104504 (2010); published online Epub09/07/ (

44. I. Goncharenko, M. I. Eremets, M. Hanfland, J. S. Tse, M. Amboage, Y. Yao, I. A. Trojan, Pressure-Induced Hydrogen-Dominant Metallic State in Aluminum Hydride. Physical Review Letters 100, 045504 (2008); published online Epub01/29/ (

45. I. Errea, M. Calandra, F. Mauri, Anharmonic free energies and phonon dispersions from the stochastic self-consistent harmonic approximation: Application to platinum and palladium hydrides. Physical Review B 89, 064302 (2014); published online Epub02/10/ ( 
46. I. Errea, M. Calandra, F. Mauri, Huge anharmonic effects in superconducting hydrides and transition metal dichalcogenides. physica status solidi (b), n/a-n/a (2014)10.1002/pssb.201451164).

47. I. Errea, B. Rousseau, A. Bergara, Enhanced Anharmonicity Under Pressure. Journal of Physics: Conference Series 377, 012060 (2012).

48. E. Wigner, H. B. Huntington, On the Possibility of a Metallic Modification of Hydrogen. J. Chem. Phys. 3, 764-770 (1935).

49. J. M. McMahon, D. M. Ceperley, High-temperature superconductivity in atomic metallic hydrogen. Physical Review B 84, 144515 (2011); published online Epub10/17/ (

50. A. W. Overhauser, Crystal structure of lithium beryllium hydride. Physical Review B 35, 411-414 (1987); published online Epub01/01/ (

51. X.-J. Chen, C. Zhang, Y. Meng, R.-Q. Zhang, H.-Q. Lin, V. V. Struzhkin, H.-k. Mao, $\beta$-tin $\rightarrow$ Imma $\rightarrow$ sh Phase Transitions of Germanium. Physical Review Letters 106, 135502 (2011); published online Epub03/30/ (

52. G. Gao, A. R. Oganov, A. Bergara, M. Martinez-Canales, T. Cui, T. Iitaka, Y. Ma, G. Zou, Superconducting High Pressure Phase of Germane. Physical Review Letters 101, 107002 (2008).

53. X.-J. Chen, J.-L. Wang, V. V. Struzhkin, H.-k. Mao, R. J. Hemley, H.-Q. Lin, Superconducting Behavior in Compressed Solid $\mathrm{SiH}_{-}\{4\}$ with a Layered Structure. Physical Review Letters 101, 077002 (2008).

54. C. J. Pickard, R. J. Needs, High-Pressure Phases of Silane. Physical Review Letters 97, 045504 (2006); published online Epub07/27/ (

55. J. Feng, W. Grochala, T. Jaroń, R. Hoffmann, A. Bergara, N. W. Ashcroft, Structures and Potential Superconductivity in $\mathrm{SiH} 4$ at High Pressure: En Route to "Metallic Hydrogen". Physical Review Letters 96, 017006 (2006); published online Epub01/11/ (

56. Y. Yao, J. S. Tse, Y. Ma, K. Tanaka, Superconductivity in high-pressure SiH 4. EPL (Europhysics Letters) 78, 37003 (2007).

57. X.-J. Chen, V. V. Struzhkin, Y. Song, A. F. Goncharov, M. Ahart, Z. Liu, H.-k. Mao, R. J. Hemley, Pressure-induced metallization of silane. Proceedings of the National Academy of Sciences 105, 20-23 (2008).

58. D. Y. Kim, R. H. Scheicher, S. Lebègue, J. Prasongkit, B. Arnaud, M. Alouani, R. Ahuja, Crystal structure of the pressure-induced metallic phase of SiH4 from ab initio theory. Proceedings of the National Academy of Sciences 105, 16454-16459 (2008); published online EpubOctober 28, 2008 (10.1073/pnas.0804148105).

59. M. Martinez-Canales, A. R. Oganov, Y. Ma, Y. Yan, A. O. Lyakhov, A. Bergara, Novel Structures and Superconductivity of Silane under Pressure. Physical Review Letters 102, 087005 (2009).

60. G. Gao, A. R. Oganov, P. Li, Z. Li, H. Wang, T. Cui, Y. Ma, A. Bergara, A. O. Lyakhov, T. Iitaka, G. Zou, High-pressure crystal structures and superconductivity of Stannane (SnH4). Proceedings of the National Academy of Sciences 107, 1317-1320 (2010); published online EpubJanuary 26, 2010 (10.1073/pnas.0908342107). 
61. O. Degtyareva, M. M. Canales, A. Bergara, X.-J. Chen, Y. Song, V. V. Struzhkin, H.-k. Mao, R. J. Hemley, Crystal structure of $\mathrm{SiH}_{4}$ at high pressure. Physical Review B 76, 064123 (2007); published online Epub08/31/ (

62. O. Degtyareva, J. E. Proctor, C. L. Guillaume, E. Gregoryanz, M. Hanfland, Formation of transition metal hydrides at high pressures. Solid State Communications 149, 1583-1586 (2009); published online Epub10// (http://dx.doi.org/10.1016/j.ssc.2009.07.022).

63. M. Hanfland, J. E. Proctor, C. L. Guillaume, O. Degtyareva, E. Gregoryanz, High-Pressure Synthesis, Amorphization, and Decomposition of Silane. Physical Review Letters 106, 095503 (2011); published online Epub03/02/ (

64. T. A. Strobel, A. F. Goncharov, C. T. Seagle, Z. Liu, M. Somayazulu, V. V. Struzhkin, R. J. Hemley, High-pressure study of silane to $150 \mathrm{GPa}$. Physical Review B 83, 144102 (2011).

65. T. Muramatsu, V. V. Struzhkin, unpublished.

66. Y. Yao, D. D. Klug, Silane plus molecular hydrogen as a possible pathway to metallic hydrogen. Proceedings of the National Academy of Sciences 107, 20893 20898 (2010); published online EpubDecember 7, 2010 (10.1073/pnas.1006508107).

67. X. Jin, X. Meng, Z. He, Y. Ma, B. Liu, T. Cui, G. Zou, H.-k. Mao, Superconducting high-pressure phases of disilane. Proceedings of the National Academy of Sciences 107, 9969-9973 (2010).

68. Y. Li, G. Gao, Y. Xie, Y. Ma, T. Cui, G. Zou, Superconductivity at $\sim 100 \mathrm{~K}$ in dense $\mathrm{SiH} 4(\mathrm{H} 2) 2$ predicted by first principles. Proceedings of the National Academy of Sciences 107, 15708-15711 (2010); published online EpubSeptember 7, 2010 (10.1073/pnas.1007354107).

69. J. A. Flores-Livas, M. Amsler, T. J. Lenosky, L. Lehtovaara, S. Botti, M. A. L. Marques, S. Goedecker, High-Pressure Structures of Disilane and Their Superconducting Properties. Physical Review Letters 108, 117004 (2012).

70. P. Zaleski-Ejgierd, R. Hoffmann, N. W. Ashcroft, High Pressure Stabilization and Emergent Forms of $\mathrm{PbH}_{4}$. Physical Review Letters 107, 037002 (2011); published online Epub07/11/ (

71. J. Hooper, E. Zurek, Rubidium Polyhydrides Under Pressure: Emergence of the Linear H3- Species. Chemistry - A European Journal 18, 5013-5021 (2012)10.1002/chem.201103205).

72. H. Wang, J. S. Tse, K. Tanaka, T. Iitaka, Y. Ma, Superconductive sodalite-like clathrate calcium hydride at high pressures. Proceedings of the National Academy of Sciences 109, 6463-6466 (2012).

73. D. C. Lonie, J. Hooper, B. Altintas, E. Zurek, Metallization of magnesium polyhydrides under pressure. Physical Review B 87, 054107 (2013).

74. C. A. Coulson, The electronic structure of H3+ Proc. Camb. Phil. Soc. 31, 244259 (1935).

75. W. Grochala, R. Hoffmann, J. Feng, N. W. Ashcroft, The Chemical Imagination at Work in Very Tight Places. Angewandte Chemie International Edition 46, 3620-3642 (2007)10.1002/anie.200602485). 
76. M. Ayouz, O. Dulieu, R. Guérout, J. Robert, V. Kokoouline, Potential energy and dipole moment surfaces of H3- molecule. The Journal of Chemical Physics 132, (2010)doi:http://dx.doi.org/10.1063/1.3424847).

77. F. Maseras, A. Lledós, E. Clot, O. Eisenstein, Transition Metal Polyhydrides: $\square$ From Qualitative Ideas to Reliable Computational Studies $\uparrow$. Chemical Reviews 100, 601-636 (2000); published online Epub2000/02/01 (10.1021/cr980397d).

78. W. Wang, A. K. Belyaev, Y. Xu, A. Zhu, C. Xiao, X.-F. Yang, Observations of H3 - and D3 - from dielectric barrier discharge plasmas. Chemical Physics Letters 377, 512-518 (2003); published online Epub8/22/ (http://dx.doi.org/10.1016/S0009-2614(03)01210-7).

79. D. A. Mazziotti, Large-Scale Semidefinite Programming for Many-Electron Quantum Mechanics. Physical Review Letters 106, 083001 (2011); published online Epub02/23/ (

80. L. Stella, C. Attaccalite, S. Sorella, A. Rubio, Strong electronic correlation in the hydrogen chain: A variational Monte Carlo study. Physical Review B 84, 245117 (2011); published online Epub12/14/ (

81. S. J. Grabowski, R. Hoffmann, Stabilizing H3-: Or Are We Stabilizing a Proton? ChemPhysChem 13, 2286-2288 (2012)10.1002/cphc.201200182).

82. M. Somayazulu, P. Dera, A. F. Goncharov, S. A. Gramsch, P. Liermann, W. Yang, Z. Liu, H.-k. Mao, R. J. Hemley, Pressure-induced bonding and compound formation in xenon-hydrogen solids. Nat Chem 2, 50-53

(2010)http://www.nature.com/nchem/journal/v2/n1/abs/nchem.445.html supplementary-information).

83. Z. G. Bazhanova, O. A. R., G. O., Fe-C and Fe-H systems at pressures of the Earth's inner core. Physics-Uspekhi 55, 489 (2012).

84. T. Scheler, M. Marqués, Z. Konôpková, C. L. Guillaume, R. T. Howie, E. Gregoryanz, High-Pressure Synthesis and Characterization of Iridium Trihydride. Physical Review Letters 111, 215503 (2013); published online Epub11/19/ (

85. R. T. Howie, O. Narygina, C. L. Guillaume, S. Evans, E. Gregoryanz, Highpressure synthesis of lithium hydride. Physical Review B 86, 064108 (2012); published online Epub08/16/ (

86. V. V. Struzhkin, D.-Y. Kim, E. Stavrou, T. Muramatsu, H.-k. Mao, C. J. Pickard, R. J. Needs, V. B. Prakapenka, A. F. Goncharov, Synthesis of Sodium Polyhydrides at High Pressures. eprint arXiv:1412.1542, (2014).

87. A. P. Drozdov, M. I. Eremets, I. A. Troyan, Conventional superconductivity at $190 \mathrm{~K}$ at high pressures. arXiv:1412.0460 [cond-mat.supr-con], (2014).

88. D. Duan, Y. Liu, F. Tian, D. Li, X. Huang, Z. Zhao, H. Yu, B. Liu, W. Tian, T. Cui, Pressure-induced metallization of dense (H2S)2H2 with high-Tc superconductivity. Sci. Rep. 4, (2014); published online Epub11/10/online (10.1038/srep06968

http://www.nature.com/srep/2014/141110/srep06968/abs/srep06968.html supplementary-information).

89. R. T. Howie, C. L. Guillaume, T. Scheler, A. F. Goncharov, E. Gregoryanz, Mixed Molecular and Atomic Phase of Dense Hydrogen. Physical Review Letters 108, 125501 (2012); published online Epub03/19/ ( 
90. A. F. Goncharov, J. S. Tse, H. Wang, J. Yang, V. V. Struzhkin, R. T. Howie, E. Gregoryanz, Bonding, structures, and band gap closure of hydrogen at high pressures. Physical Review B 87, 024101 (2013); published online Epub01/02/ (

91. C.-s. Zha, Z. Liu, M. Ahart, R. Boehler, R. J. Hemley, High-Pressure Measurements of Hydrogen Phase IV Using Synchrotron Infrared Spectroscopy. Physical Review Letters 110, 217402 (2013); published online Epub05/21/ (

92. R. T. Howie, T. Scheler, C. L. Guillaume, E. Gregoryanz, Proton tunneling in phase IV of hydrogen and deuterium. Physical Review B 86, 214104 (2012); published online Epub12/10/ (

93. R. T. Howie, I. B. Magdău, A. F. Goncharov, G. J. Ackland, E. Gregoryanz, Phonon Localization by Mass Disorder in Dense Hydrogen-Deuterium Binary Alloy. Physical Review Letters 113, 175501 (2014); published online Epub10/21/

94. C.-s. Zha, R. E. Cohen, H.-k. Mao, R. J. Hemley, Raman measurements of phase transitions in dense solid hydrogen and deuterium to $325 \mathrm{GPa}$. Proceedings of the National Academy of Sciences 111, $4792-4797$ (2014); published online EpubApril 1, 2014 (10.1073/pnas.1402737111).

95. L. Gao, Y. Y. Xue, F. Chen, Q. Xiong, R. L. Meng, D. Ramirez, C. W. Chu, J. H. Eggert, H. K. Mao, Superconductivity up to $164 \mathrm{~K}$ in HaBa2Cam$1 \mathrm{CumO} 2 \mathrm{~m}+2+\mathrm{d}(\mathrm{m}=1,2$, and 3$)$ under quasihydrostatic pressures. Phys. Rev. B 50, 4260-4263 (1994).

96. M. I. Eremets, I. A. Troyan, Conductive dense hydrogen. Nat Mater 10, 927-931 (2011); published online Epub12//print (http://www.nature.com/nmat/journal/v10/n12/abs/nmat3175.html supplementary-information).

97. W. Nellis, I. Silvera, Has Metallic Hydrogen Been Made in a Diamond Anvil Cell? arXiv.org > cond-mat > arXiv:1201.0407, (2013).

98. P. Cudazzo, G. Profeta, A. Sanna, A. Floris, A. Continenza, S. Massidda, E. K. U. Gross, Electron-phonon interaction and superconductivity in metallic molecular hydrogen. I. Electronic and dynamical properties under pressure. Physical Review B 81, 134505 (2010); published online Epub04/02/ (

99. P. Cudazzo, G. Profeta, A. Sanna, A. Floris, A. Continenza, S. Massidda, E. K. U. Gross, Electron-phonon interaction and superconductivity in metallic molecular hydrogen. II. Superconductivity under pressure. Physical Review B 81, 134506 (2010); published online Epub04/02/ (

100. L. Dubrovinsky, N. Dubrovinskaia, V. B. Prakapenka, A. M. Abakumov, Implementation of micro-ball nanodiamond anvils for high-pressure studies above 6[thinsp]Mbar. Nat Commun 3, 1163

(2012)http://www.nature.com/ncomms/journal/v3/n10/suppinfo/ncomms2160_S1 .html).

101. C. R. Rotundu, T. Ćuk, R. L. Greene, Z.-X. Shen, R. J. Hemley, V. V. Struzhkin, High-pressure resistivity technique for quasi-hydrostatic compression experiments. Review of Scientific Instruments 84, (2013)doi:http://dx.doi.org/10.1063/1.4809025).

102. V. E. Antonov, T. E. Antonova, I. T. Belash, V. I. Rashupkin, Superconductivities of high-pressure phases in the metal-hydrogen systems. High Pressure Research 
1, 315-320 (1989); published online Epub1989/11/01 (10.1080/08957958908202487).

103. Y. Li, J. Hao, H. Liu, Y. Li, Y. Ma, The metallization and superconductivity of dense hydrogen sulfide. The Journal of Chemical Physics 140, (2014)doi:http://dx.doi.org/10.1063/1.4874158).

104. D. R. Krahn, R. L. Henry, D. B. Tanner, P. E. Wigen, The critical fields of superconducting palladium hydride. physica status solidi (a) 46, 209-212 (1978)10.1002/pssa.2210460125).

105. T. Skośkiewicz, Superconductivity in the palladium-hydrogen system. physica status solidi (b) 59, 329-334 (1973)10.1002/pssb.2220590133).

106. D. S. McLachlan, T. B. Doyle, J. P. Burger, The superconducting properties of PdHx $\lesssim$ 1. J Low Temp Phys 26, 589-601 (1977); published online Epub1977/02/01 (10.1007/BF00655432).

107. N. E. Alekseevskii, Y. A. Samarskii, H. Wolf, V. I. Tserbo, V. M. Zakosarenko, Critical magnetic fields of superconducting palladium hydride. JETP Lett. 19, 676-679 (1974).

108. A. Eichler, H. Wühl, B. Stritzker, Tunneling experiments on superconducting palladium-deuterium alloys. Solid State Communications 17, 213-216 (1975); published online Epub7/15/ (http://dx.doi.org/10.1016/0038-1098(75)90045-9).

109. P. J. Silverman, C. V. Briscoe, Superconducting tunneling in PdH. Physics Letters A 53, 221-222 (1975); published online Epub6/16/ (http://dx.doi.org/10.1016/0375-9601(75)90414-4).

110. J. Igalson, L. Sniadower, A. J. Pindor, T. Skoskiewicz, K. Blüthner, F. Dettmann, Tunneling observation of the superconducting energy gap in the palladium hydride thin films. Solid State Communications 17, 309-312 (1975); published online Epub8/1/ (http://dx.doi.org/10.1016/0038-1098(75)90300-2).

111. H. Hemmes, A. Driessen, R. Griessen, M. Gupta, Isotope effects and pressure dependence of the Tc of superconducting stoichiometric PdH and PdD synthesized and measured in a diamond anvil cell. Physical Review B 39, 41104118 (1989); published online Epub03/01/ (

112. M. Dietrich, W. Gey, H. Rietschel, C. B. Satterthwaite, Pressure dependence of the superconducting transition temperature of Th4H15. Solid State Communications 15, 941-943 (1974); published online Epub9/1/ (http://dx.doi.org/10.1016/0038-1098(74)90699-1).

113. J. F. Miller, R. H. Caton, C. B. Satterthwaite, Low-temperature heat capacity of normal and superconducting thorium hydride and thorium deuteride. Physical Review B 14, 2795-2800 (1976); published online Epub10/01/ (

114. V. E. Antonov, I. T. Belash, O. V. Zharikov, A. I. Latynin, A. V. Pal'nichenko, Superconductivity of molybdenum hydride and deuteride. Fizika Tverdogo Tela (in Russian) 30, 598-600 (1988). 